\title{
Robustness of DEWMA versus EWMA Control Charts to Non-Normal Processes
}

Saad Saeed Alkahtani

Performance Measurement Center of Government Agencies, Institute of Public Administration, Riyadh, Saudi Arabia

Follow this and additional works at: http://digitalcommons.wayne.edu/jmasm

Part of the Applied Statistics Commons, Social and Behavioral Sciences Commons, and the Statistical Theory Commons

\section{Recommended Citation}

Alkahtani, Saad Saeed (2013) "Robustness of DEWMA versus EWMA Control Charts to Non-Normal Processes," Journal of Modern Applied Statistical Methods: Vol. 12 : Iss. 1 , Article 18.

DOI: $10.22237 /$ jmasm/1367381820

Available at: http://digitalcommons.wayne.edu/jmasm/vol12/iss1/18

This Regular Article is brought to you for free and open access by the Open Access Journals at DigitalCommons@WayneState. It has been accepted for inclusion in Journal of Modern Applied Statistical Methods by an authorized editor of DigitalCommons@WayneState. 


\title{
Robustness of DEWMA versus EWMA Control Charts to Non-Normal Processes
}

\author{
Saad Saeed Alkahtani \\ Performance Measurement Center of Government Agencies, \\ Institute of Public Administration \\ Riyadh, Saudi Arabia
}

Exponentially weighted moving average (EWMA) and double EWMA (DEWMA) control charts were designed under the normality assumption. This study considers various skewed (Gamma) and symmetric non-normal (t) distributions to examine the effect of non-normality on the average run length (ARL) performance of EWMA and DEWMA. ARL performances were investigated and compared using Monte Carlo simulations. Results show that DEWMA charts can be designed to be robust to non-normality, that the ARL performances of EWMA and DEWMA charts were more robust to $t$ distributions and DEWMA was more robust to non-normality for larger values of the smoothing parameter.

Key words: Average run length, control charts, EWMA, DEWMA, robustness, non-normality.

Introduction

A popular control chart used to detect small shifts in a process mean is the EWMA (Roberts, 1959). In an effort to increase the sensitivity of EWMA control charts to detect small shifts and drifts in a process, a double EWMA (DEWMA) control chart was developed by Shamma and Shamma (1992). Zhang (2002) has conducted extensive studies on DEWMA control charts for the mean.

Like most commonly used control charts, the traditional EWMA and DEWMA control charts for monitoring process means were developed under the assumption of normality. The behavior of the EWMA control chart performance for non-normal distributions has been investigated. Borror, et al. (1999) used the Markov chain method and simulations to study the average run length (ARL) performance of the EWMA control charts for the mean of

Saad Saeed Alkahtani is an Assistant Professor of Applied Statistics. Dr. Alkahtani teaches courses in statistics such as SPC, regression, multivariate statistics, experimental design, SAS and SPSS. He also provides consultations in statistics and has published several articles. Email him at: alkahtanisas@yahoo.com. skewed (gamma) and heavy-tailed ( $\mathrm{t}$ ) symmetric non-normal distributions. They concluded that the ARL performance of a well-designed EWMA control chart was robust to violations of the normality assumption.

As a part of an extensive study of the effect of non-normality and auto-correlation on the performance of EWMA control charts, Stoumbos and Reynolds (2000) concluded that some combinations of EWMA control charts for detecting small shifts in a process mean and/or variance can be designed to be robust to the violation of normality assumption. Montgomery (2005) found that an appropriately designed EWMA chart will perform well even for nonnormal data.

Simulation studies on the robustness of an EWMA control chart for process mean monitoring have been conducted by Borror, et al. (1999) and by Koshti and Kalgonda (2011). In addition, Human, et al. (2011) ran an extensive simulation to study the robustness of an EWMA control chart for individual observations. They investigated the in-control robustness of the designs studied by Borror, et al. (1999) and found that, with some types of non-normal data, caution should be taken not to overuse EWMA charts.

Studies related to the concept of robustness to non-normality of the EWMA control statistic have also been conducted. For 
example, Lin and Chou (2010) investigated the robustness of EWMA and -EWMA control charts with variable sampling intervals to nonnormality; Shiau and Hsu (2005) studied the robustness of the EWMA control chart to nonnormality for auto-correlated process; and Calzada and Scariano (2003) investigated the robustness of the MaxEWMA control charts to the violation of normality. The robustness to non-normality of DEWMA control charts for detecting shifts in a process mean has not been investigated; thus, this study considers the robustness of DEWMA and compares it to EWMA using Monte Carlo simulations.

Background

If $X_{i}$, where $i=1,2, \ldots, m$, is a sequence of random variables taken from a normal distribution with mean $\mu_{0}$ and variance $\sigma^{2}$, then the EWMA control statistic is defined as:

$$
Z_{i}=\lambda X_{i}+(1-\lambda) Z_{i-1}
$$

where $0<\lambda \leq 1$ is a smoothing parameter and $Z_{0}=\mu_{0}$ (i.e., the in-control or target process mean). Typically, $\mu_{0}$ is unknown and is estimated from the preliminary sample by the sample mean $\bar{X}$. The control limits for the EWMA control chart are:

$$
\begin{aligned}
& U C L=\mu_{0}+L \sigma \sqrt{\left(\frac{\lambda}{2-\lambda}\right)\left[1-(1-\lambda)^{2 i}\right]} \\
& C L=\mu_{0} \\
& L C L=\mu_{0}-L \sigma \sqrt{\left(\frac{\lambda}{2-\lambda}\right)\left[1-(1-\lambda)^{2 i}\right]}
\end{aligned}
$$

where $\mathrm{L}$ is the distance between the control limits and the center line (CL) measured in $\sigma$ units. For large values of $i$, the control limits become:

$$
\begin{aligned}
& U C L=\mu_{0}+L \sigma \sqrt{\left(\frac{\lambda}{2-\lambda}\right)} \\
& C L=\mu_{0} \\
& L C L=\mu_{0}-L \sigma \sqrt{\left(\frac{\lambda}{2-\lambda}\right)}
\end{aligned}
$$

The DEWMA control statistic $Z_{i}$ is defined as:

$$
\begin{aligned}
& Y_{i}=\lambda X_{i}+(1-\lambda) Y_{i-1} \\
& Z_{i}=\lambda Y_{i}+(1-\lambda) Z_{i-1}
\end{aligned}
$$

such that $0<\lambda<1$ and $Y_{0}=Z_{0}=\mu_{0}$. It can be shown that (see Appendix A)

$$
\begin{gathered}
Z_{i}=\lambda^{2} \sum_{j=1}^{i}(i-j+1)(1-\lambda)^{i-j} X_{j} \\
+i \lambda(1-\lambda)^{i} Y_{0}+(1-\lambda)^{i} Z_{0} \\
E\left(Z_{i}\right)=\mu_{0}
\end{gathered}
$$

and

$$
\begin{gathered}
1+(1-\lambda)^{2}-\left(i^{2}+2 i+1\right)(1-\lambda)^{2 i} \\
\sigma_{Z_{i}}^{2}=\lambda^{4} \frac{+\left(2 i^{2}+2 i-1\right)(1-\lambda)^{2 i+2}-i^{2}(1-\lambda)^{2 i+4}}{\left(1-(1-\lambda)^{2}\right)^{3}} \sigma_{0}^{2}
\end{gathered}
$$

The control limits for the DEWMA control chart are: 
$U C L=$

$\mu_{0}+L \sigma \sqrt{\begin{array}{c}1+(1-\lambda)^{2}-\left(i^{2}+2 i+1\right)(1-\lambda)^{2 i} \\ \lambda^{4} \frac{+\left(2 i^{2}+2 i-1\right)(1-\lambda)^{2 i+2}-i^{2}(1-\lambda)^{2 i+4}}{\left(1-(1-\lambda)^{2}\right)^{3}}\end{array}}$

$C L=\mu_{0}$

$L C L=$

$\mu_{0}-L \sigma \sqrt{\begin{array}{c}1+(1-\lambda)^{2}-\left(i^{2}+2 i+1\right)(1-\lambda)^{2 i} \\ \lambda^{4} \frac{+\left(2 i^{2}+2 i-1\right)(1-\lambda)^{2 i+2}-i^{2}(1-\lambda)^{2 i+4}}{\left(1-(1-\lambda)^{2}\right)^{3}}\end{array}}$

where $\mathrm{L}$ is as defined. For large values of $i$, the control limits become:

$$
\begin{aligned}
& U C L=\mu_{0}+L \sigma \sqrt{\frac{\lambda\left(2-2 \lambda+\lambda^{2}\right)}{(2-\lambda)^{3}}} \\
& C L=\mu_{0} \\
& L C L=\mu_{0}-L \sigma \sqrt{\frac{\lambda\left(2-2 \lambda+\lambda^{2}\right)}{(2-\lambda)^{3}}}
\end{aligned}
$$

For both EWMA and DEWMA control charts, the control statistics in (1.1) and (1.4), respectively, are plotted on the control chart and the process is considered to be out of control if the plotted point lies outside the LCL and UCL.

Borror, et al. (1999) used various symmetric and skewed non-normal distributions to study the robustness of the EWMA control charts for process mean. They considered the $t$ distributions with different numbers of degrees of freedom (i.e., $d f=4,6,8,10,15,20,30,40$, 50). The mean and the variance of the $t$ distribution are: $\mu=0$ and $\sigma^{2}=\frac{d f}{d f-2}$ such that $d f>2$, respectively. For the skewed distribution, the authors considered Gamma distributions denoted $\operatorname{Gam}(\alpha, \beta)$ with $\alpha=4$,
$3,2,1,0.5$ and $\beta=1$. The mean and variance of the gamma distribution are: $\mu=\frac{\alpha}{\beta}$ and $\sigma^{2}=\frac{\alpha}{\beta^{2}}$, respectively

Average Run Length Performances of EWMA vs DEWMA

This study considers $t$ distributions with $d f=4,10,20,40$, and 50 and $\operatorname{Gam}(\alpha, \beta)$ with $\alpha=4,3,2,1,0.5$ and $\beta=1$ to compare the effect of non-normality on the RL performance of both EWMA and DEWMA control charts. For $\lambda=0.10,0.20,0.30,0.40,0.50$, the values of $\mathrm{L}$ for both control charts were chosen such that the in-control ARL $\cong 370.4$ (as used by Borror, et al. 1999) when the process followed a normal distribution. As shown herein, the robustness study of EWMA charts reproduces Borror's findings and they were considered here for the ease of comparison.

\section{Methodology}

All RL calculations were completed based on 10,000 Monte Carlo simulations for each scenario, using SAS ${ }^{\circledR}$ V. 9.2 RANNOR and RANGAM functions. The simulations were conducted as follows:

1. Pseudo random numbers from normal, gamma, and $t$ distributions were generated by SAS ${ }^{\circledR}$ V. 9.2 RANNOR and RANGAM functions.

2. The control statistics $Y_{i}$ for EWMA and $Z_{i}$ for DEWMA were computed.

3. The control statistic was compared with an experimental LCL and UCL and a run length was obtained and recorded.

4. After 10,000 simulation runs, the mean of the 10, 000 derived RL (ARL) and the standard error of ARL (SEARL) values were obtained. 
5. The values of $\mathrm{L}$ were chosen such that the computed in-control ALR is almost equal to 370.4 .

Steps from 1 to 4 were run for each scenario of the combination of the previously assigned different values of the parameters $\lambda, \alpha, \beta, d f$ and shifts.

Tables 1 and 2 summarize the results of the calculated $\mathrm{L}$ values and the in-control ARL along with their SEARL in parenthesis for EWMA and DEWMA control chart for Normal and Various Gamma and $t$ distributions. The following are noted:

i. For Gamma and $t$ distributions, the incontrol ARL for both EWMA and DEWMA were reasonably close to 370.4 for small values of $\lambda$ (i.e., $\lambda<0.20$ ) especially with larger $\alpha$ and $d f$ values of gamma and $t$ distributions, respectively (i.e., when both distributions approach normality). ii. The degree of in-control ARL deterioration for both EWMA and DEWMA was less for $t$ distribution than for gamma distribution.

In general, for gamma and $t$ distributions with larger parameters $\alpha, \beta$ and $d f$ respectively, the in-control ARL values for EWMA were better (larger) than those for DEWMA for small values of $\lambda(\lambda<0.20)$. Conversely, the in-control ARL values for DEWMA were better (i.e., larger) than those for EWMA for larger values of $\lambda(\lambda>0.40)$ particularly with gamma distribution.

Tables 3 and 4 show the out-of-control ARLs for the EWMA and DEWMA Control Charts for Various Gamma Distributions and shifts in the mean measured in standard deviation units. For small shifts in the process mean (shift $=0.25$ ) and $\lambda>0.20$, the out-ofcontrol ARLs for both EWMA and DEWMA are significantly less than the value that would be obtained if the process was normal; other than

Table 1: In-control ARL for EWMA and DEWMA Control Chart for Various Normal and Various Gamma Distributions

\begin{tabular}{|c|c|c|c|c|c|c|c|c|c|c|}
\cline { 2 - 11 } \multicolumn{1}{c|}{} & \multicolumn{5}{c|}{$\begin{array}{c}\text { EWMA } \\
\text { (SEARL) }\end{array}$} & \multicolumn{5}{c|}{$\begin{array}{c}\text { DEWMA } \\
\text { (SEARL) }\end{array}$} \\
\hline$\lambda$ & 0.1 & 0.2 & 0.3 & 0.4 & 0.5 & 0.1 & 0.2 & 0.3 & 0.4 & 0.5 \\
\hline L & 2.698 & 2.856 & 2.929 & 2.956 & 2.977 & 3.796 & 4.018 & 4.069 & 4.059 & 3.991 \\
\hline \multirow{2}{*}{ Normal } & 369.9 & 370.3 & 370.2 & 370.2 & 368.9 & 370.2 & 370.6 & 370.9 & 369.8 & 369.9 \\
& $(3.62)$ & $(3.64)$ & $(3.67)$ & $(3.71)$ & $(3.71)$ & $(3.65)$ & $(3.75)$ & $(3.68)$ & $(3.60)$ & $(3.66)$ \\
\hline \multirow{2}{*}{$\operatorname{Gam}(4,1)$} & 339.8 & 261.5 & 202.7 & 159.9 & 135.8 & 330.2 & $\begin{array}{c}259.4 \\
(2.59)\end{array}$ & $\begin{array}{c}200.2 \\
(2.00)\end{array}$ & $\begin{array}{c}162.8 \\
(1.64)\end{array}$ & $\begin{array}{c}139.7 \\
(1.40)\end{array}$ \\
\hline \multirow{2}{*}{$\operatorname{Gam}(3,1)$} & $(3.32)$ & $(2.56)$ & $(2.02)$ & $(1.57)$ & $(1.37)$ & $(3.31)$ & $(2.5)$ & \\
& 332.6 & 238.9 & 182.6 & 143.3 & 121.8 & 326.5 & 239.2 & 176.0 & 140.2 & 121.3 \\
& $(3.21)$ & $(2.38)$ & $(1.82)$ & $(1.41)$ & $(1.21)$ & $(3.22)$ & $(2.39)$ & $(1.74)$ & $(1.38)$ & $(1.18)$ \\
\hline \multirow{2}{*}{$\operatorname{Gam}(2,1)$} & 316.4 & 206.3 & 149.7 & 123.2 & 101.4 & 297.3 & 205.7 & 150.0 & 120.4 & 103.1 \\
& $(3.06)$ & $(2.02)$ & $(1.51)$ & $(1.23)$ & $(1.01)$ & $(2.91)$ & $(2.00)$ & $(1.48)$ & $(1.19)$ & $(1.04)$ \\
\hline \multirow{2}{*}{$\operatorname{Gam}(1,1)$} & 271.4 & 163.2 & 117.6 & 92.8 & 77.2 & 264.0 & 162.7 & 117.3 & 91.0 & 77.8 \\
& $(2.69)$ & $(1.61)$ & $(1.17)$ & $(0.91)$ & $(0.78)$ & $(2.63)$ & $(1.63)$ & $(1.17)$ & $(0.91)$ & $(0.78)$ \\
\hline \multirow{2}{*}{$\operatorname{Gam}(0.5,1)$} & 228.9 & 133.9 & 94.6 & 75.8 & 62.6 & 228.0 & 133.6 & 93.7 & 74.9 & 64.1 \\
& $(2.21)$ & $(1.32)$ & $(0.92)$ & $(0.75)$ & $(0.63)$ & $(2.32)$ & $(1.30)$ & $(0.95)$ & $(0.72)$ & $(0.64)$ \\
\hline
\end{tabular}


that, the ARLs are comparable. Generally, the behavior of robustness to gamma distributions of both charts was similar.

Tables 5 and 6 show out-of-control ARL's for the EWMA and DEWMA Control Charts for Various $t$ Distributions and shifts. The ARL performance of EWMA and DEWMA for both $t$ and normal distributions are comparable except for the case that shift $=0.25$ and $\mathrm{df}<20$; that is, the difference between the ALRs of both normal and $t$ distribution is considerable. For the in-control case, the degree of out-of-control ARL deterioration for both EWMA and DEWMA is less for $t$ distribution than for gamma distribution.

\section{Conclusion}

The effect of non-normality on the ARL performances for EWMA and DEWMA was investigated using Monte Carlo simulations. SAS ${ }^{8} \quad$ V. 9.2 RANNOR and RANGAM functions were used to generate data from various normal, gamma, and $t$ distributions and to perform the calculations for all scenarios.
Results show that, in general, the incontrol ARL performances of both EWMA and DEWMA control charts were more robust for the $t$ distribution than for gamma. The degree of robustness of the EWMA and DEWMA control charts to non-normality increased for smaller values of smoothing parameter and as the $t$ and gamma distributions approach normality. In addition, for gamma and $t$ distributions, the incontrol ARL values for EWMA were more robust than those for DEWMA for small values of $\lambda(\lambda<0.20)$; however, the in-control ARL values for DEWMA were more robust than those for EWMA for large values of $\lambda(\lambda>0.40)$ specifically with gamma distribution with larger parameters

Similarly to the in-control case, the outof-control ARLs of EWMA and DEWMA were more robust for $t$ distribution than for gamma. However, some details needed to be considered. It was noticed that the out-of-control ARL for EWMA and DEWMA was significantly less for gamma comparing to normal-theory ARL for small shift (shift $=0.25)$ and large smoothing

Table 2: In-control ARL for EWMA and DEWMA Control Chart for Various Normal and Various $t$ Distributions

\begin{tabular}{|c|c|c|c|c|c|c|c|c|c|c|}
\cline { 2 - 11 } \multicolumn{1}{c|}{} & \multicolumn{5}{c|}{$\begin{array}{c}\text { EWMA } \\
\text { (SEARL) }\end{array}$} & \multicolumn{5}{c|}{$\begin{array}{c}\text { DEWMA } \\
\text { (SEARL) }\end{array}$} \\
\hline$\lambda$ & 0.1 & 0.2 & 0.3 & 0.4 & 0.5 & 0.1 & 0.2 & 0.3 & 0.4 & 0.5 \\
\hline $\mathrm{L}$ & 2.698 & 2.856 & 2.929 & 2.956 & 2.977 & 3.796 & 4.018 & 4.069 & 4.059 & 3.991 \\
\hline \multirow{2}{*}{ Normal } & 369.9 & 370.3 & 370.2 & 370.2 & 368.9 & 370.2 & 370.6 & 370.9 & 369.8 & 369.9 \\
& $(3.62)$ & $(3.64)$ & $(3.67)$ & $(3.71)$ & $(3.71)$ & $(3.65)$ & $(3.75)$ & $(3.68)$ & $(3.60)$ & $(3.66)$ \\
\hline \multirow{2}{*}{$t_{50}$} & 365.3 & 340.3 & 354.3 & 329.6 & 314.6 & 349.1 & 348.6 & 332.8 & 336.0 & 307.2 \\
& $(3.48)$ & $(3.31)$ & $(3.46)$ & $(3.25)$ & $(3.06)$ & $(3.46)$ & $(3.51)$ & $(3.33)$ & $(3.36)$ & $(3.06)$ \\
\hline \multirow{2}{*}{$t_{40}$} & 358.3 & 349.8 & 338.6 & 310.8 & 306.4 & 344.6 & 350.7 & 328.7 & 314.0 & 302.4 \\
& $(3.46)$ & $(3.44)$ & $(3.31)$ & $(3.09)$ & $(3.09)$ & $(3.40)$ & $(3.48)$ & $(3.21)$ & $(3.21)$ & $(3.02)$ \\
\hline \multirow{2}{*}{$t_{20}$} & 351.8 & 330.7 & 303.5 & 266.9 & 251.8 & 340.9 & 322.4 & 298.3 & 268.0 & 251.5 \\
& $(3.47)$ & $(3.19)$ & $(2.97)$ & $(2.62)$ & $(2.58)$ & $(3.32)$ & $(3.19)$ & $(2.92)$ & $(2.65)$ & $(2.58)$ \\
\hline \multirow{2}{*}{$t_{10}$} & 331.2 & 283.7 & 244.4 & 201.7 & 175.2 & 327.1 & 276.0 & 233.7 & 204.5 & 180.0 \\
& $(3.24)$ & $(2.82)$ & $(2.43)$ & $(2.00)$ & $(1.75)$ & $(3.19)$ & $(2.72)$ & $(2.29)$ & $(2.05)$ & $(1.75)$ \\
\hline \multirow{2}{*}{$t_{4}$} & 268.6 & 187.5 & 148.2 & 117.4 & 102.2 & 264.3 & 191.6 & 141.6 & 118.1 & 101.5 \\
& $(2.62)$ & $(1.82)$ & $(1.47)$ & $(1.16)$ & $(1.02)$ & $(2.60)$ & $(1.90)$ & $(1.38)$ & $(1.19)$ & $(1.01)$ \\
\hline
\end{tabular}


value $(\lambda>0.20)$ and comparable otherwise. For the $t$ distribution, the out-of-control ARL for EWMA and DEWMA were comparable to the normal-theory ARL except for shift $=0.25$ and $d f<20$. In addition, for larger values of $\lambda$ the out-of-control ARLs for DEWMA were similar or slightly better than those for EWMA. Shamma and Shamma (1992) stated:

Baxley (1990) reported EWMA results for a simulated industrial process requiring a larger $\lambda(\lambda=0.35)$ but the optimal EWMA chart requires that $\lambda=0.05$. dEWMA charts will be more sensitive to cases which can be best modeled by dEWMA models with larger values as compared to EWMA since such values will be non-optimal for EWMA charts (p. 21).

Based on both the results of this study and Shamma and Shamma's report, DEWMA control charts should be considered in practice because the EWMA is non-optimal for larger values of $\lambda$. Also, the variability of the simulated average run length for DEWMA is generally smaller than that for EWMA. These properties should motivate the use of DEWMA in industrial process.

\section{References}

Baxley, R. V. (1990). Discussion. Technometrics, 32, 13-16.

Borror, C. M., Montgomery, D. C., \& Runger, G. C. (1999). Robustness of the EWMA control chart to non-normality. Journal of Quality Technology, 10, 139-149.

Calzada, M. E., \& Scariano, S. M. (2003). The robustness of the MaxEWMA chart to non-normality. Communications in StatisticsSimulations and Computations, 32(2), 573-590.

Gradshteyn, I. S., \& Ryzhik, I. M. (1979). Table of integrals, series, and products. Waltham, MA: Academic Press.
Human, S. W., Kritzinger, P., \& Chakraborti, S. (2011). Robustness of the EWMA control chart for individual observations. Journal of Applied Statistics, 38(10), 2071-2087.

Koshti, V. V., \& Kalgonda, A. A. (2011). A study of robustness of the exponentially weighted moving average control chart: a simulation approach. International Journal of Advanced Scientific and technical research, 2(1), 519-525.

Lin, Y. C., \& Chou, C. Y. (2010). Robustness of EWMA and combined $\bar{X}$ EWMA control charts with variable sampling intervals to non-normality. Journal of Applied Statistics, 38(3), 553-570.

Montgomery, D. C. (2005). Introduction to statistical quality control $\left(5^{\text {th }} E d\right.$.). New York, NY: John Wiley \& Sons, Inc.

Roberts, S. W. (1959). Control charts tests based on geometric moving average. Technimetrics, 1(3), 239-250.

Shamma, S. E., \& Shamma, A. K. (1992). Development and evaluation of control charts using double exponentially weighted moving averages. International Journal of Quality \& Reliability Management, $9(6), 18-25$.

Shiau, J. H., \& Hsu, Y. C. (2005). Robustness of the EWMA control chart to nonnormality for autocorrelated processes. Quality Technology \& Quantitative Management, 2(2), 125-146.

Stoumbos, Z. G., \& Reynolds, M. R. (2000). Robustness to non-normality and autocorrelation of individuals control charts.

Journal of Statistical Computation and Simulation, 66(2), 145-187.

Zhang, L. Y. (2002). EWMA control charts and extended EWMA control charts. Unpublished doctoral dissertation. University of Regina, Saskatchewan, Canada. 
Table 3: Out-of-control ARL's for the EWMA Control Charts for Normal and Various Gamma Distributions

\begin{tabular}{|c|c|c|c|c|c|c|c|c|}
\hline & \multirow[b]{2}{*}{ Distribution } & \multicolumn{7}{|c|}{$\begin{array}{c}\text { Shift } \\
\text { (Number of Standard Deviations) }\end{array}$} \\
\hline & & 0.25 & 0.50 & 1.00 & 1.50 & 2.00 & 2.50 & 3.00 \\
\hline \multirow{6}{*}{$\begin{array}{c}\text { EWMA } \\
\lambda=0.1 \\
L= \\
2.698\end{array}$} & Normal & $\begin{array}{c}90.4 \\
(0.80)\end{array}$ & $\begin{array}{c}27.9 \\
(0.19)\end{array}$ & $\begin{array}{c}9.6 \\
(0.04)\end{array}$ & $\begin{array}{c}5.8 \\
(0.02)\end{array}$ & $\begin{array}{c}4.2 \\
(0.01)\end{array}$ & $\begin{array}{c}3.3 \\
(0.01)\end{array}$ & $\begin{array}{c}2.8 \\
(0.01)\end{array}$ \\
\hline & $\operatorname{Gam}(4,1)$ & $\begin{array}{l}78.8 \\
(0.72)\end{array}$ & $\begin{array}{c}27.9 \\
(0.20)\end{array}$ & $\begin{array}{l}10.0 \\
(0.05)\end{array}$ & $\begin{array}{c}5.9 \\
(0.02)\end{array}$ & $\begin{array}{c}4.2 \\
(0.01)\end{array}$ & $\begin{array}{c}3.3 \\
(0.01)\end{array}$ & $\begin{array}{c}2.8 \\
(0.01)\end{array}$ \\
\hline & $\operatorname{Gam}(3,1)$ & $\begin{array}{c}76.6 \\
(0.69)\end{array}$ & $\begin{array}{l}28.5 \\
(0.21)\end{array}$ & $\begin{array}{l}10.0 \\
(0.05)\end{array}$ & $\begin{array}{c}5.9 \\
(0.02)\end{array}$ & $\begin{array}{c}4.2 \\
(0.01)\end{array}$ & $\begin{array}{c}3.3 \\
(0.01)\end{array}$ & $\begin{array}{c}2.8 \\
(0.01)\end{array}$ \\
\hline & $\operatorname{Gam}(2,1)$ & $\begin{array}{c}76.2 \\
(0.67)\end{array}$ & $\begin{array}{c}29.2 \\
(0.22)\end{array}$ & $\begin{array}{c}10.1 \\
(0.05)\end{array}$ & $\begin{array}{c}5.9 \\
(0.02)\end{array}$ & $\begin{array}{c}4.2 \\
(0.01) \\
\end{array}$ & $\begin{array}{c}3.3 \\
(0.01)\end{array}$ & $\begin{array}{c}2.8 \\
(0.01)\end{array}$ \\
\hline & $\operatorname{Gam}(1,1)$ & $\begin{array}{l}74.5 \\
(0.68)\end{array}$ & $\begin{array}{c}30.0 \\
(0.23)\end{array}$ & $\begin{array}{c}10.4 \\
(0.05)\end{array}$ & $\begin{array}{c}5.9 \\
(0.02)\end{array}$ & $\begin{array}{c}4.2 \\
(0.01)\end{array}$ & $\begin{array}{c}3.3 \\
(0.01)\end{array}$ & $\begin{array}{c}2.8 \\
(0.01)\end{array}$ \\
\hline & $\operatorname{Gam}(.5,1)$ & $\begin{array}{c}73.4 \\
(0.67)\end{array}$ & $\begin{array}{l}30.5 \\
(0.24)\end{array}$ & $\begin{array}{c}10.7 \\
(0.05)\end{array}$ & $\begin{array}{c}6.0 \\
(0.02)\end{array}$ & $\begin{array}{c}4.2 \\
(0.01)\end{array}$ & $\begin{array}{c}3.3 \\
(0.01)\end{array}$ & $\begin{array}{c}2.7 \\
(0.01)\end{array}$ \\
\hline \multirow{6}{*}{$\begin{array}{c}\text { EWMA } \\
\lambda=0.2 \\
L= \\
2.856\end{array}$} & Normal & $\begin{array}{c}119.3 \\
(1.17)\end{array}$ & $\begin{array}{c}36.0 \\
(0.30)\end{array}$ & $\begin{array}{c}9.8 \\
(0.06)\end{array}$ & $\begin{array}{c}5.2 \\
(0.02)\end{array}$ & $\begin{array}{c}3.6 \\
(0.01)\end{array}$ & $\begin{array}{c}2.8 \\
(0.01)\end{array}$ & $\begin{array}{c}2.3 \\
(0.01)\end{array}$ \\
\hline & $\operatorname{Gam}(4,1)$ & $\begin{array}{c}82.0 \\
(0.80)\end{array}$ & $\begin{array}{c}33.2 \\
(0.29)\end{array}$ & $\begin{array}{c}10.2 \\
(0.06)\end{array}$ & $\begin{array}{c}5.4 \\
(0.02)\end{array}$ & $\begin{array}{c}3.7 \\
(0.01)\end{array}$ & $\begin{array}{c}2.8 \\
(0.01)\end{array}$ & $\begin{array}{c}2.3 \\
(0.01)\end{array}$ \\
\hline & $\operatorname{Gam}(3,1)$ & $\begin{array}{c}79.7 \\
(0.77)\end{array}$ & $\begin{array}{c}32.8 \\
(0.29)\end{array}$ & $\begin{array}{c}10.2 \\
(0.07)\end{array}$ & $\begin{array}{c}5.4 \\
(0.02)\end{array}$ & $\begin{array}{c}3.6 \\
(0.01)\end{array}$ & $\begin{array}{c}2.8 \\
(0.01)\end{array}$ & $\begin{array}{c}2.3 \\
(0.01)\end{array}$ \\
\hline & $\operatorname{Gam}(2,1)$ & $\begin{array}{c}74.7 \\
(0.71) \\
\end{array}$ & $\begin{array}{c}32.4 \\
(0.29) \\
\end{array}$ & $\begin{array}{c}10.3 \\
(0.29) \\
\end{array}$ & $\begin{array}{c}5.5 \\
(0.07) \\
\end{array}$ & $\begin{array}{c}3.7 \\
(0.03) \\
\end{array}$ & $\begin{array}{c}2.8 \\
(0.01)\end{array}$ & $\begin{array}{c}2.3 \\
(0.01)\end{array}$ \\
\hline & $\operatorname{Gam}(1,1)$ & $\begin{array}{c}67.4 \\
(0.65)\end{array}$ & $\begin{array}{c}32.0 \\
(0.28)\end{array}$ & $\begin{array}{c}10.8 \\
(0.07)\end{array}$ & $\begin{array}{c}5.7 \\
(0.03) \\
\end{array}$ & $\begin{array}{c}3.7 \\
(0.01)\end{array}$ & $\begin{array}{c}2.8 \\
(0.01)\end{array}$ & $\begin{array}{c}2.3 \\
(0.01)\end{array}$ \\
\hline & $\operatorname{Gam}(.5,1)$ & $\begin{array}{c}62.7 \\
(0.60)\end{array}$ & $\begin{array}{c}32.8 \\
(0.29)\end{array}$ & $\begin{array}{c}11.8 \\
(0.08)\end{array}$ & $\begin{array}{c}5.8 \\
(0.03)\end{array}$ & $\begin{array}{c}3.6 \\
(0.01)\end{array}$ & $\begin{array}{c}2.8 \\
(0.01)\end{array}$ & $\begin{array}{c}2.3 \\
(0.01)\end{array}$ \\
\hline \multirow{6}{*}{$\begin{array}{c}\text { EWMA } \\
\lambda=0.3 \\
\mathrm{~L}= \\
2.929\end{array}$} & Normal & $\begin{array}{c}149.4 \\
(1.46)\end{array}$ & $\begin{array}{c}46.8 \\
(0.44)\end{array}$ & $\begin{array}{c}10.9 \\
(0.08)\end{array}$ & $\begin{array}{c}5.2 \\
(0.03)\end{array}$ & $\begin{array}{c}3.4 \\
(0.01)\end{array}$ & $\begin{array}{c}2.6 \\
(0.01)\end{array}$ & $\begin{array}{c}2.1 \\
(0.01)\end{array}$ \\
\hline & $\operatorname{Gam}(4,1)$ & $\begin{array}{c}79.1 \\
(0.76)\end{array}$ & $\begin{array}{c}36.1 \\
(0.34)\end{array}$ & $\begin{array}{c}11.2 \\
(0.08)\end{array}$ & $\begin{array}{c}5.5 \\
(0.03)\end{array}$ & $\begin{array}{c}3.5 \\
(0.01)\end{array}$ & $\begin{array}{c}2.6 \\
(0.01)\end{array}$ & $\begin{array}{c}2.1 \\
(0.01)\end{array}$ \\
\hline & $\operatorname{Gam}(3,1)$ & $\begin{array}{c}72.9 \\
(0.72) \\
\end{array}$ & $\begin{array}{c}35.9 \\
(0.32)\end{array}$ & $\begin{array}{c}11.4 \\
(0.09)\end{array}$ & $\begin{array}{c}5.6 \\
(0.03)\end{array}$ & $\begin{array}{c}3.5 \\
(0.01) \\
\end{array}$ & $\begin{array}{c}2.6 \\
(0.01) \\
\end{array}$ & $\begin{array}{c}2.1 \\
(0.01)\end{array}$ \\
\hline & $\operatorname{Gam}(2,1)$ & $\begin{array}{c}68.7 \\
(0.67)\end{array}$ & $\begin{array}{c}33.5 \\
(0.31)\end{array}$ & $\begin{array}{c}11.6 \\
(0.09)\end{array}$ & $\begin{array}{c}5.7 \\
(0.03)\end{array}$ & $\begin{array}{c}3.6 \\
(0.01)\end{array}$ & $\begin{array}{c}2.6 \\
(0.01)\end{array}$ & $\begin{array}{c}2.1 \\
(0.01)\end{array}$ \\
\hline & $\operatorname{Gam}(1,1)$ & $\begin{array}{c}60.4 \\
(0.59)\end{array}$ & $\begin{array}{c}33.1 \\
(0.32)\end{array}$ & $\begin{array}{c}12.2 \\
(0.10)\end{array}$ & $\begin{array}{c}5.9 \\
(0.03)\end{array}$ & $\begin{array}{c}3.6 \\
(0.01)\end{array}$ & $\begin{array}{c}2.6 \\
(0.01)\end{array}$ & $\begin{array}{c}2.1 \\
(0.01)\end{array}$ \\
\hline & $\operatorname{Gam}(.5,1)$ & $\begin{array}{c}56.3 \\
(0.55)\end{array}$ & $\begin{array}{c}32.4 \\
(0.30)\end{array}$ & $\begin{array}{c}13.1 \\
(0.10)\end{array}$ & $\begin{array}{c}6.4 \\
(0.04)\end{array}$ & $\begin{array}{c}3.6 \\
(0.01)\end{array}$ & $\begin{array}{c}2.6 \\
(0.01)\end{array}$ & $\begin{array}{c}2.1 \\
(0.01)\end{array}$ \\
\hline
\end{tabular}


Table 3 (continued): Out-of-control ARL's for the EWMA Control Charts for Normal and Various Gamma Distributions

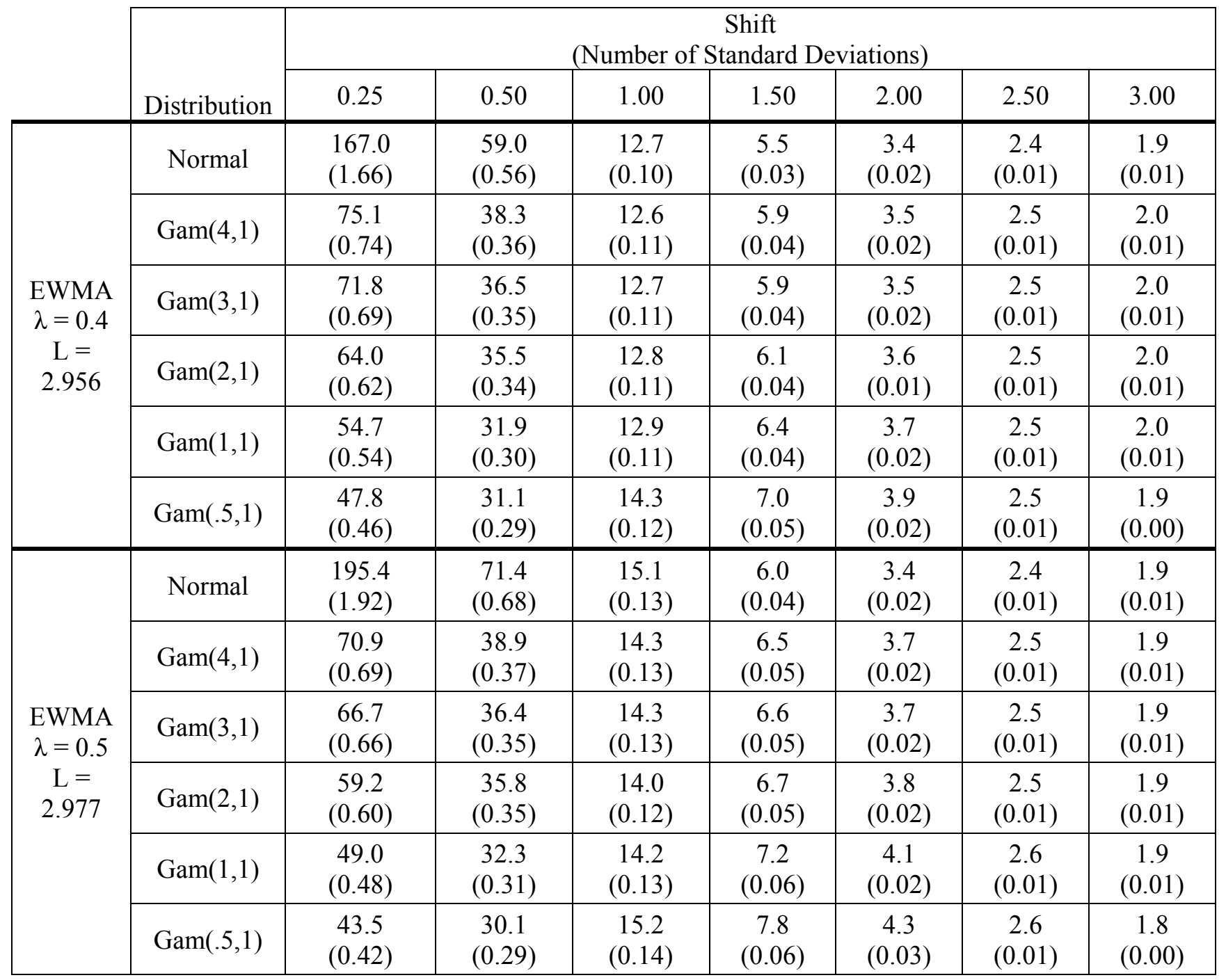


Table 4: Out-of-control ARL's for the DEWMA Control Charts for Normal and Various Gamma Distributions

\begin{tabular}{|c|c|c|c|c|c|c|c|c|}
\hline & \multirow[b]{2}{*}{ Distribution } & \multicolumn{7}{|c|}{$\begin{array}{c}\text { Shift } \\
\text { (Number of Standard Deviations) }\end{array}$} \\
\hline & & 0.25 & 0.50 & 1.00 & 1.50 & 2.00 & 2.50 & 3.00 \\
\hline \multirow{6}{*}{$\begin{array}{c}\text { DEWMA } \\
\lambda=0.1 \\
\mathrm{~L}=3.796\end{array}$} & Normal & $\begin{array}{c}88.2 \\
(0.80) \\
\end{array}$ & $\begin{array}{c}27.5 \\
(0.20) \\
\end{array}$ & $\begin{array}{c}9.7 \\
(0.04) \\
\end{array}$ & $\begin{array}{c}5.7 \\
(0.02) \\
\end{array}$ & $\begin{array}{c}4.2 \\
(0.01) \\
\end{array}$ & $\begin{array}{c}3.3 \\
(0.01)\end{array}$ & $\begin{array}{c}2.7 \\
(0.01) \\
\end{array}$ \\
\hline & $\operatorname{Gam}(4,1)$ & $\begin{array}{l}78.3 \\
(0.69)\end{array}$ & $\begin{array}{c}28.4 \\
(0.20)\end{array}$ & $\begin{array}{l}10.0 \\
(0.05)\end{array}$ & $\begin{array}{c}5.9 \\
(0.02)\end{array}$ & $\begin{array}{c}4.2 \\
(0.01)\end{array}$ & $\begin{array}{c}3.3 \\
(0.01)\end{array}$ & $\begin{array}{c}2.8 \\
(0.01)\end{array}$ \\
\hline & $\operatorname{Gam}(3,1)$ & $\begin{array}{l}75.3 \\
(0.66)\end{array}$ & $\begin{array}{c}28.8 \\
(0.21)\end{array}$ & $\begin{array}{c}10.1 \\
(0.05)\end{array}$ & $\begin{array}{c}5.9 \\
(0.02)\end{array}$ & $\begin{array}{c}4.2 \\
(0.01)\end{array}$ & $\begin{array}{c}3.3 \\
(0.01)\end{array}$ & $\begin{array}{c}2.8 \\
(0.01)\end{array}$ \\
\hline & $\operatorname{Gam}(2,1)$ & $\begin{array}{l}75.3 \\
(0.69)\end{array}$ & $\begin{array}{c}28.7 \\
(0.22)\end{array}$ & $\begin{array}{c}10.1 \\
(0.05)\end{array}$ & $\begin{array}{c}5.9 \\
(0.02)\end{array}$ & $\begin{array}{c}4.2 \\
(0.01)\end{array}$ & $\begin{array}{c}3.3 \\
(0.01)\end{array}$ & $\begin{array}{c}2.7 \\
(0.01)\end{array}$ \\
\hline & $\operatorname{Gam}(1,1)$ & $\begin{array}{c}73.9 \\
(0.68) \\
\end{array}$ & $\begin{array}{r}29.9 \\
(0.23) \\
\end{array}$ & $\begin{array}{c}10.4 \\
(0.05)\end{array}$ & $\begin{array}{c}5.9 \\
(0.02) \\
\end{array}$ & $\begin{array}{c}4.2 \\
(0.01) \\
\end{array}$ & $\begin{array}{c}3.3 \\
(0.01) \\
\end{array}$ & $\begin{array}{c}2.7 \\
(0.01)\end{array}$ \\
\hline & $\operatorname{Gam}(.5,1)$ & $\begin{array}{c}71.6 \\
(0.67)\end{array}$ & $\begin{array}{c}30.4 \\
(0.24)\end{array}$ & $\begin{array}{c}10.7 \\
(0.05)\end{array}$ & $\begin{array}{c}5.9 \\
(0.02)\end{array}$ & $\begin{array}{c}4.2 \\
(0.01)\end{array}$ & $\begin{array}{c}3.3 \\
(0.01)\end{array}$ & $\begin{array}{c}2.7 \\
(0.00)\end{array}$ \\
\hline \multirow{6}{*}{$\begin{array}{c}\text { DEWMA } \\
\lambda=0.2 \\
L=4.018\end{array}$} & Normal & $\begin{array}{l}122.7 \\
(1.16)\end{array}$ & $\begin{array}{c}36.6 \\
(0.31)\end{array}$ & $\begin{array}{c}9.7 \\
(0.06)\end{array}$ & $\begin{array}{c}5.3 \\
(0.02)\end{array}$ & $\begin{array}{c}3.6 \\
(0.01)\end{array}$ & $\begin{array}{c}2.8 \\
(0.01)\end{array}$ & $\begin{array}{c}2.3 \\
(0.01)\end{array}$ \\
\hline & $\operatorname{Gam}(4,1)$ & $\begin{array}{c}81.1 \\
(0.78) \\
\end{array}$ & $\begin{array}{c}33.1 \\
(0.29) \\
\end{array}$ & $\begin{array}{c}10.4 \\
(0.06) \\
\end{array}$ & $\begin{array}{c}5.4 \\
(0.02)\end{array}$ & $\begin{array}{c}3.6 \\
(0.01)\end{array}$ & $\begin{array}{c}2.8 \\
(0.01)\end{array}$ & $\begin{array}{c}2.3 \\
(0.01)\end{array}$ \\
\hline & $\operatorname{Gam}(3,1)$ & $\begin{array}{c}79.9 \\
(0.77)\end{array}$ & $\begin{array}{c}33.0 \\
(0.29)\end{array}$ & $\begin{array}{c}10.3 \\
(0.06)\end{array}$ & $\begin{array}{c}5.4 \\
(0.03)\end{array}$ & $\begin{array}{c}3.7 \\
(0.01)\end{array}$ & $\begin{array}{c}2.8 \\
(0.01)\end{array}$ & $\begin{array}{c}2.3 \\
(0.01)\end{array}$ \\
\hline & $\operatorname{Gam}(2,1)$ & $\begin{array}{c}74.6 \\
(0.71) \\
\end{array}$ & $\begin{array}{c}32.5 \\
(0.29) \\
\end{array}$ & $\begin{array}{c}10.6 \\
(0.07) \\
\end{array}$ & $\begin{array}{c}5.5 \\
(0.02) \\
\end{array}$ & $\begin{array}{c}3.7 \\
(0.01) \\
\end{array}$ & $\begin{array}{c}2.8 \\
(0.01) \\
\end{array}$ & $\begin{array}{c}2.3 \\
(0.01) \\
\end{array}$ \\
\hline & $\operatorname{Gam}(1,1)$ & $\begin{array}{c}67.9 \\
(0.64)\end{array}$ & $\begin{array}{c}32.9 \\
(0.29)\end{array}$ & $\begin{array}{c}11.0 \\
(0.07)\end{array}$ & $\begin{array}{c}5.7 \\
(0.03)\end{array}$ & $\begin{array}{c}3.7 \\
(0.01)\end{array}$ & $\begin{array}{c}2.8 \\
(0.01)\end{array}$ & $\begin{array}{c}2.3 \\
(0.01)\end{array}$ \\
\hline & $\operatorname{Gam}(.5,1)$ & $\begin{array}{c}63.6 \\
(0.60)\end{array}$ & $\begin{array}{c}32.6 \\
(0.30)\end{array}$ & $\begin{array}{c}11.6 \\
(0.08)\end{array}$ & $\begin{array}{c}5.9 \\
(0.03)\end{array}$ & $\begin{array}{c}3.7 \\
(0.01)\end{array}$ & $\begin{array}{c}2.8 \\
(0.01)\end{array}$ & $\begin{array}{c}2.3 \\
(0.01)\end{array}$ \\
\hline \multirow{6}{*}{$\begin{array}{c}\text { DEWMA } \\
\lambda=0.3 \\
L=4.069\end{array}$} & Normal & $\begin{array}{l}149.2 \\
(1.44)\end{array}$ & $\begin{array}{c}46.0 \\
(0.42)\end{array}$ & $\begin{array}{c}10.9 \\
(0.08)\end{array}$ & $\begin{array}{c}5.2 \\
(0.03)\end{array}$ & $\begin{array}{c}3.4 \\
(0.01)\end{array}$ & $\begin{array}{c}2.5 \\
(0.01)\end{array}$ & $\begin{array}{c}2.1 \\
(0.01)\end{array}$ \\
\hline & $\operatorname{Gam}(4,1)$ & $\begin{array}{c}78.8 \\
(0.76)\end{array}$ & $\begin{array}{c}36.7 \\
(0.33)\end{array}$ & $\begin{array}{c}11.4 \\
(0.09)\end{array}$ & $\begin{array}{c}5.5 \\
(0.3)\end{array}$ & $\begin{array}{c}3.5 \\
(0.01)\end{array}$ & $\begin{array}{c}2.6 \\
(0.01)\end{array}$ & $\begin{array}{c}2.1 \\
(0.01)\end{array}$ \\
\hline & $\operatorname{Gam}(3,1)$ & $\begin{array}{c}74.2 \\
(0.71)\end{array}$ & $\begin{array}{c}35.4 \\
(0.32)\end{array}$ & $\begin{array}{c}11.3 \\
(0.08)\end{array}$ & $\begin{array}{c}5.5 \\
(0.03)\end{array}$ & $\begin{array}{c}3.5 \\
(0.01)\end{array}$ & $\begin{array}{c}2.6 \\
(0.01)\end{array}$ & $\begin{array}{c}2.1 \\
(0.01)\end{array}$ \\
\hline & $\operatorname{Gam}(2,1)$ & $\begin{array}{c}68.5 \\
(0.65) \\
\end{array}$ & $\begin{array}{c}33.9 \\
(0.32) \\
\end{array}$ & $\begin{array}{c}11.4 \\
(0.09) \\
\end{array}$ & $\begin{array}{c}5.6 \\
(0.03) \\
\end{array}$ & $\begin{array}{c}3.5 \\
(0.01) \\
\end{array}$ & $\begin{array}{c}2.6 \\
(0.01) \\
\end{array}$ & $\begin{array}{c}2.1 \\
(0.01) \\
\end{array}$ \\
\hline & $\operatorname{Gam}(1,1)$ & $\begin{array}{c}60.1 \\
(0.57)\end{array}$ & $\begin{array}{c}32.8 \\
(0.31)\end{array}$ & $\begin{array}{c}12.1 \\
(0.09)\end{array}$ & $\begin{array}{c}5.9 \\
(0.03)\end{array}$ & $\begin{array}{c}3.6 \\
(0.01)\end{array}$ & $\begin{array}{c}2.6 \\
(0.01)\end{array}$ & $\begin{array}{c}2.1 \\
(0.01)\end{array}$ \\
\hline & $\operatorname{Gam}(.5,1)$ & $\begin{array}{c}54.7 \\
(0.54) \\
\end{array}$ & $\begin{array}{c}32.9 \\
(0.32) \\
\end{array}$ & $\begin{array}{c}13.2 \\
(0.11) \\
\end{array}$ & $\begin{array}{c}6.3 \\
(0.04) \\
\end{array}$ & $\begin{array}{c}3.6 \\
(0.01) \\
\end{array}$ & $\begin{array}{c}2.6 \\
(0.01) \\
\end{array}$ & $\begin{array}{c}2.0 \\
(0.01) \\
\end{array}$ \\
\hline
\end{tabular}


Table 4 (continued): Out-of-control ARL's for the DEWMA Control Charts for Normal and Various Gamma Distributions

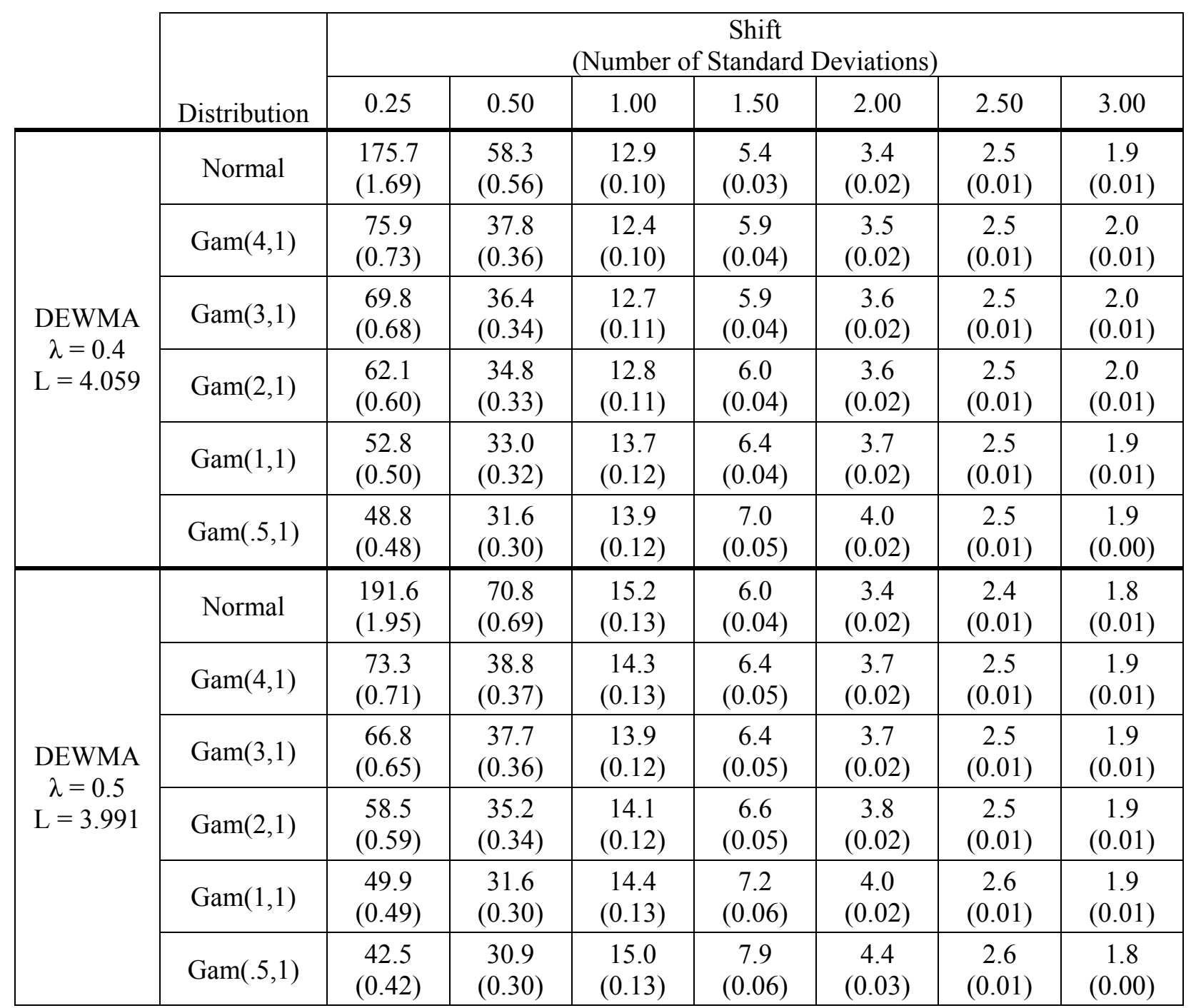


DEWMA VS. EWMA CONTROL CHART ROBUSTNESS TO NON-NORMAL PROCESSES

Table 5: Out-of-control ARL's for the EWMA Control Charts for Normal and Various t Distributions

\begin{tabular}{|c|c|c|c|c|c|c|c|c|}
\hline & \multirow[b]{2}{*}{ Distribution } & \multicolumn{7}{|c|}{$\begin{array}{c}\text { Shift } \\
\text { (Number of Standard Deviations) }\end{array}$} \\
\hline & & 0.25 & 0.50 & 1.00 & 1.50 & 2.00 & 2.50 & 3.00 \\
\hline \multirow{6}{*}{$\begin{array}{c}\text { EWMA } \\
\lambda=0.1 \\
L= \\
2.698\end{array}$} & Normal & $\begin{array}{c}90.4 \\
(0.80)\end{array}$ & $\begin{array}{l}27.9 \\
(0.19)\end{array}$ & $\begin{array}{c}9.6 \\
(0.04)\end{array}$ & $\begin{array}{c}9.6 \\
(0.04)\end{array}$ & $\begin{array}{c}4.2 \\
(0.01)\end{array}$ & $\begin{array}{c}3.3 \\
(0.01)\end{array}$ & $\begin{array}{c}2.8 \\
(0.01)\end{array}$ \\
\hline & $t_{50}$ & $\begin{array}{c}87.4 \\
(0.78)\end{array}$ & $\begin{array}{c}27.9 \\
(0.19)\end{array}$ & $\begin{array}{c}9.8 \\
(0.04)\end{array}$ & $\begin{array}{c}9.8 \\
(0.04)\end{array}$ & $\begin{array}{c}4.2 \\
(0.01)\end{array}$ & $\begin{array}{c}3.3 \\
(0.01)\end{array}$ & $\begin{array}{c}2.8 \\
(0.01)\end{array}$ \\
\hline & $t_{40}$ & $\begin{array}{c}88.9 \\
(0.78)\end{array}$ & $\begin{array}{c}28.5 \\
(0.20)\end{array}$ & $\begin{array}{c}9.7 \\
(0.04)\end{array}$ & $\begin{array}{c}9.7 \\
(0.04)\end{array}$ & $\begin{array}{c}4.2 \\
(0.01)\end{array}$ & $\begin{array}{c}3.3 \\
(0.01)\end{array}$ & $\begin{array}{c}2.8 \\
(0.01)\end{array}$ \\
\hline & $t_{20}$ & $\begin{array}{c}90.7 \\
(0.81)\end{array}$ & $\begin{array}{c}28.5 \\
(0.20)\end{array}$ & $\begin{array}{c}9.7 \\
(0.04)\end{array}$ & $\begin{array}{c}9.7 \\
(0.04)\end{array}$ & $\begin{array}{c}4.2 \\
(0.01)\end{array}$ & $\begin{array}{c}3.3 \\
(0.01)\end{array}$ & $\begin{array}{c}2.8 \\
(0.01)\end{array}$ \\
\hline & $t_{10}$ & $\begin{array}{c}90.4 \\
(0.80)\end{array}$ & $\begin{array}{c}28.3 \\
(0.20)\end{array}$ & $\begin{array}{c}9.7 \\
(0.05)\end{array}$ & $\begin{array}{c}9.7 \\
(0.05)\end{array}$ & $\begin{array}{c}4.2 \\
(0.01)\end{array}$ & $\begin{array}{c}3.3 \\
(0.01)\end{array}$ & $\begin{array}{c}2.8 \\
(0.01)\end{array}$ \\
\hline & $t_{4}$ & $\begin{array}{l}94.6 \\
(0.86)\end{array}$ & $\begin{array}{c}30.4 \\
(0.22)\end{array}$ & $\begin{array}{c}9.8 \\
(0.04)\end{array}$ & $\begin{array}{c}9.8 \\
(0.04)\end{array}$ & $\begin{array}{c}4.2 \\
(0.01)\end{array}$ & $\begin{array}{c}3.3 \\
(0.01)\end{array}$ & $\begin{array}{c}2.8 \\
(0.01)\end{array}$ \\
\hline \multirow{6}{*}{$\begin{array}{c}\text { EWMA } \\
\lambda=0.2 \\
L= \\
2.856\end{array}$} & Normal & $\begin{array}{l}119.3 \\
(1.17)\end{array}$ & $\begin{array}{c}36.0 \\
(0.30)\end{array}$ & $\begin{array}{c}9.8 \\
(0.06)\end{array}$ & $\begin{array}{c}9.8 \\
(0.06)\end{array}$ & $\begin{array}{c}3.6 \\
(0.01)\end{array}$ & $\begin{array}{c}2.8 \\
(0.01)\end{array}$ & $\begin{array}{c}2.3 \\
(0.01)\end{array}$ \\
\hline & $t_{50}$ & $\begin{array}{c}117.4 \\
(1.10)\end{array}$ & $\begin{array}{c}35.9 \\
(0.30)\end{array}$ & $\begin{array}{c}9.8 \\
(0.06)\end{array}$ & $\begin{array}{c}9.8 \\
(0.06)\end{array}$ & $\begin{array}{c}3.6 \\
(0.01)\end{array}$ & $\begin{array}{c}2.8 \\
(0.01)\end{array}$ & $\begin{array}{c}2.3 \\
(0.01)\end{array}$ \\
\hline & $t_{40}$ & $\begin{array}{l}120.8 \\
(1.16)\end{array}$ & $\begin{array}{c}36.6 \\
(0.31)\end{array}$ & $\begin{array}{c}9.7 \\
(0.06)\end{array}$ & $\begin{array}{c}9.7 \\
(0.06)\end{array}$ & $\begin{array}{c}3.6 \\
(0.01)\end{array}$ & $\begin{array}{c}2.8 \\
(0.01)\end{array}$ & $\begin{array}{c}2.3 \\
(0.01)\end{array}$ \\
\hline & $t_{20}$ & $\begin{array}{l}119.6 \\
(1.13)\end{array}$ & $\begin{array}{c}36.3 \\
(0.31)\end{array}$ & $\begin{array}{c}9.8 \\
(0.06)\end{array}$ & $\begin{array}{c}9.8 \\
(0.06)\end{array}$ & $\begin{array}{c}3.6 \\
(0.01)\end{array}$ & $\begin{array}{c}2.8 \\
(0.01)\end{array}$ & $\begin{array}{c}2.3 \\
(0.01)\end{array}$ \\
\hline & $t_{10}$ & $\begin{array}{l}113.3 \\
(1.07)\end{array}$ & $\begin{array}{c}37.2 \\
(0.32)\end{array}$ & $\begin{array}{c}10.0 \\
(0.06)\end{array}$ & $\begin{array}{c}10.0 \\
(0.06)\end{array}$ & $\begin{array}{c}3.6 \\
(0.01)\end{array}$ & $\begin{array}{c}2.8 \\
(0.01)\end{array}$ & $\begin{array}{c}2.3 \\
(0.01)\end{array}$ \\
\hline & $t_{4}$ & $\begin{array}{c}104.1 \\
(1.00)\end{array}$ & $\begin{array}{c}40.3 \\
(0.35)\end{array}$ & $\begin{array}{c}10.2 \\
(0.06)\end{array}$ & $\begin{array}{c}10.2 \\
(0.06)\end{array}$ & $\begin{array}{c}3.6 \\
(0.01)\end{array}$ & $\begin{array}{c}2.8 \\
(0.01)\end{array}$ & $\begin{array}{c}2.3 \\
(0.01)\end{array}$ \\
\hline \multirow{6}{*}{$\begin{array}{c}\text { EWMA } \\
\lambda=0.3 \\
L= \\
2.929\end{array}$} & Normal & $\begin{array}{l}149.4 \\
(1.46)\end{array}$ & $\begin{array}{c}46.8 \\
(0.44)\end{array}$ & $\begin{array}{c}10.9 \\
(0.08)\end{array}$ & $\begin{array}{c}10.9 \\
(0.08)\end{array}$ & $\begin{array}{c}3.4 \\
(0.01)\end{array}$ & $\begin{array}{c}2.6 \\
(0.01)\end{array}$ & $\begin{array}{c}2.1 \\
(0.01)\end{array}$ \\
\hline & $t_{50}$ & $\begin{array}{l}143.7 \\
(1.43)\end{array}$ & $\begin{array}{c}47.1 \\
(0.43)\end{array}$ & $\begin{array}{c}11.0 \\
(0.08)\end{array}$ & $\begin{array}{c}11.0 \\
(0.08)\end{array}$ & $\begin{array}{c}3.4 \\
(0.01)\end{array}$ & $\begin{array}{c}2.6 \\
(0.01)\end{array}$ & $\begin{array}{c}2.1 \\
(0.01)\end{array}$ \\
\hline & $t_{40}$ & $\begin{array}{l}143.9 \\
(1.42)\end{array}$ & $\begin{array}{c}46.5 \\
(0.43)\end{array}$ & $\begin{array}{c}11.0 \\
(0.08)\end{array}$ & $\begin{array}{c}11.0 \\
(0.08)\end{array}$ & $\begin{array}{c}3.4 \\
(0.01)\end{array}$ & $\begin{array}{c}2.6 \\
(0.01)\end{array}$ & $\begin{array}{c}2.1 \\
(0.01)\end{array}$ \\
\hline & $t_{20}$ & $\begin{array}{l}141.7 \\
(1.39) \\
\end{array}$ & $\begin{array}{c}46.6 \\
(0.43) \\
\end{array}$ & $\begin{array}{c}11.0 \\
(0.08) \\
\end{array}$ & $\begin{array}{c}11.0 \\
(0.08) \\
\end{array}$ & $\begin{array}{c}3.4 \\
(0.01) \\
\end{array}$ & $\begin{array}{c}2.6 \\
(0.01) \\
\end{array}$ & $\begin{array}{c}2.1 \\
(0.01) \\
\end{array}$ \\
\hline & $t_{10}$ & $\begin{array}{l}126.5 \\
(1.24) \\
\end{array}$ & $\begin{array}{c}47.6 \\
(0.45) \\
\end{array}$ & $\begin{array}{c}11.2 \\
(0.08) \\
\end{array}$ & $\begin{array}{c}11.2 \\
(0.08)\end{array}$ & $\begin{array}{c}3.4 \\
(0.01)\end{array}$ & $\begin{array}{c}2.6 \\
(0.01)\end{array}$ & $\begin{array}{c}2.1 \\
(0.01)\end{array}$ \\
\hline & $t_{4}$ & $\begin{array}{l}105.5 \\
(1.03)\end{array}$ & $\begin{array}{c}50.8 \\
(0.47)\end{array}$ & $\begin{array}{c}12.3 \\
(0.09)\end{array}$ & $\begin{array}{c}12.3 \\
(0.09)\end{array}$ & $\begin{array}{c}3.4 \\
(0.01)\end{array}$ & $\begin{array}{c}2.5 \\
(0.01)\end{array}$ & $\begin{array}{c}2.1 \\
(0.01)\end{array}$ \\
\hline
\end{tabular}


SAAD SAEED ALKAHTANI

Table 5 (continued): Out-of-control ARL's for the EWMA Control Charts for Normal and Various t Distributions

\begin{tabular}{|c|c|c|c|c|c|c|c|c|}
\hline & \multirow[b]{2}{*}{ Distribution } & \multicolumn{7}{|c|}{$\begin{array}{c}\text { Shift } \\
\text { (Number of Standard Deviations) }\end{array}$} \\
\hline & & 0.25 & 0.50 & 1.00 & 1.50 & 2.00 & 2.50 & 3.00 \\
\hline \multirow{6}{*}{$\begin{array}{c}\text { EWMA } \\
\lambda=0.4 \\
L= \\
2.956\end{array}$} & Normal & $\begin{array}{r}167.0 \\
(1.66)\end{array}$ & $\begin{array}{c}59.0 \\
(0.56)\end{array}$ & $\begin{array}{c}12.7 \\
(0.10)\end{array}$ & $\begin{array}{c}12.7 \\
(0.10)\end{array}$ & $\begin{array}{c}3.4 \\
(0.02)\end{array}$ & $\begin{array}{c}2.4 \\
(0.01)\end{array}$ & $\begin{array}{c}1.9 \\
(0.01)\end{array}$ \\
\hline & $t_{50}$ & $\begin{array}{l}163.5 \\
(1.59) \\
\end{array}$ & $\begin{array}{c}58.2 \\
(0.55) \\
\end{array}$ & $\begin{array}{c}12.7 \\
(0.10)\end{array}$ & $\begin{array}{c}12.7 \\
(0.10)\end{array}$ & $\begin{array}{c}3.3 \\
(0.02)\end{array}$ & $\begin{array}{c}2.4 \\
(0.01)\end{array}$ & $\begin{array}{c}1.9 \\
(0.01)\end{array}$ \\
\hline & $t_{40}$ & $\begin{array}{l}160.9 \\
(1.60)\end{array}$ & $\begin{array}{l}56.7 \\
(0.55)\end{array}$ & $\begin{array}{c}12.8 \\
(0.10)\end{array}$ & $\begin{array}{c}12.8 \\
(0.10)\end{array}$ & $\begin{array}{c}3.3 \\
(0.02)\end{array}$ & $\begin{array}{c}2.4 \\
(0.01)\end{array}$ & $\begin{array}{c}2.0 \\
(0.01)\end{array}$ \\
\hline & $t_{20}$ & $\begin{array}{l}149.5 \\
(1.46)\end{array}$ & $\begin{array}{c}56.4 \\
(0.52)\end{array}$ & $\begin{array}{c}13.1 \\
(0.10)\end{array}$ & $\begin{array}{c}13.1 \\
(0.10)\end{array}$ & $\begin{array}{c}3.3 \\
(0.02)\end{array}$ & $\begin{array}{c}2.4 \\
(0.01)\end{array}$ & $\begin{array}{c}2.0 \\
(0.01)\end{array}$ \\
\hline & $t_{10}$ & $\begin{array}{c}128.4 \\
(1.28)\end{array}$ & $\begin{array}{c}54.4 \\
(0.51) \\
\end{array}$ & $\begin{array}{c}13.1 \\
(0.10)\end{array}$ & $\begin{array}{c}13.1 \\
(0.10)\end{array}$ & $\begin{array}{c}3.4 \\
(0.02)\end{array}$ & $\begin{array}{c}2.4 \\
(0.01)\end{array}$ & $\begin{array}{c}1.9 \\
(0.01)\end{array}$ \\
\hline & $t_{4}$ & $\begin{array}{r}93.3 \\
(0.93) \\
\end{array}$ & $\begin{array}{c}56.0 \\
(0.53) \\
\end{array}$ & $\begin{array}{c}15.1 \\
(0.13) \\
\end{array}$ & $\begin{array}{c}15.1 \\
(0.13) \\
\end{array}$ & $\begin{array}{r}3.3 \\
(0.01) \\
\end{array}$ & $\begin{array}{c}2.4 \\
(0.01) \\
\end{array}$ & $\begin{array}{c}2.0 \\
(0.01) \\
\end{array}$ \\
\hline \multirow{6}{*}{$\begin{array}{c}\text { EWMA } \\
\lambda=0.5 \\
L= \\
2.977\end{array}$} & Normal & $\begin{array}{l}195.4 \\
(1.92) \\
\end{array}$ & $\begin{array}{c}71.4 \\
(0.68) \\
\end{array}$ & $\begin{array}{c}15.1 \\
(0.13) \\
\end{array}$ & $\begin{array}{c}15.1 \\
(0.13) \\
\end{array}$ & $\begin{array}{r}3.4 \\
(0.02) \\
\end{array}$ & $\begin{array}{c}2.4 \\
(0.01) \\
\end{array}$ & $\begin{array}{c}1.9 \\
(0.01) \\
\end{array}$ \\
\hline & $t_{50}$ & $\begin{array}{r}182.2 \\
(1.77)\end{array}$ & $\begin{array}{c}69.0 \\
(0.67)\end{array}$ & $\begin{array}{c}15.2 \\
(0.13)\end{array}$ & $\begin{array}{c}15.2 \\
(0.13)\end{array}$ & $\begin{array}{c}3.4 \\
(0.02)\end{array}$ & $\begin{array}{c}2.4 \\
(0.01)\end{array}$ & $\begin{array}{c}1.9 \\
(0.01)\end{array}$ \\
\hline & $t_{40}$ & $\begin{array}{c}170.4 \\
(1.69)\end{array}$ & $\begin{array}{c}70.5 \\
(0.68)\end{array}$ & $\begin{array}{c}15.3 \\
(0.13)\end{array}$ & $\begin{array}{c}15.3 \\
(0.13)\end{array}$ & $\begin{array}{c}3.5 \\
(0.02)\end{array}$ & $\begin{array}{c}2.4 \\
(0.01)\end{array}$ & $\begin{array}{c}1.8 \\
(0.01)\end{array}$ \\
\hline & $t_{20}$ & $\begin{array}{c}157.1 \\
(1.54) \\
\end{array}$ & $\begin{array}{c}66.7 \\
(0.65) \\
\end{array}$ & $\begin{array}{c}15.6 \\
(0.14) \\
\end{array}$ & $\begin{array}{c}15.6 \\
(0.14) \\
\end{array}$ & $\begin{array}{c}3.5 \\
(0.02) \\
\end{array}$ & $\begin{array}{c}2.4 \\
(0.01) \\
\end{array}$ & $\begin{array}{c}1.9 \\
(0.01) \\
\end{array}$ \\
\hline & $t_{10}$ & $\begin{array}{r}131.0 \\
(1.34) \\
\end{array}$ & $\begin{array}{c}62.5 \\
(0.60) \\
\end{array}$ & $\begin{array}{c}16.0 \\
(0.14)\end{array}$ & $\begin{array}{c}16.0 \\
(0.14)\end{array}$ & $\begin{array}{c}3.4 \\
(0.02)\end{array}$ & $\begin{array}{c}2.4 \\
(0.01)\end{array}$ & $\begin{array}{c}1.9 \\
(0.01)\end{array}$ \\
\hline & $t_{4}$ & $\begin{array}{c}87.1 \\
(0.88)\end{array}$ & $\begin{array}{c}58.8 \\
(0.57)\end{array}$ & $\begin{array}{c}18.6 \\
(0.17)\end{array}$ & $\begin{array}{c}18.6 \\
(0.17)\end{array}$ & $\begin{array}{c}3.5 \\
(0.02)\end{array}$ & $\begin{array}{c}2.4 \\
(0.01)\end{array}$ & $\begin{array}{c}1.9 \\
(0.01)\end{array}$ \\
\hline
\end{tabular}


DEWMA VS. EWMA CONTROL CHART ROBUSTNESS TO NON-NORMAL PROCESSES

Table 6: Out-of-control ARL's for the DEWMA Control Charts for Normal and Various t Distributions

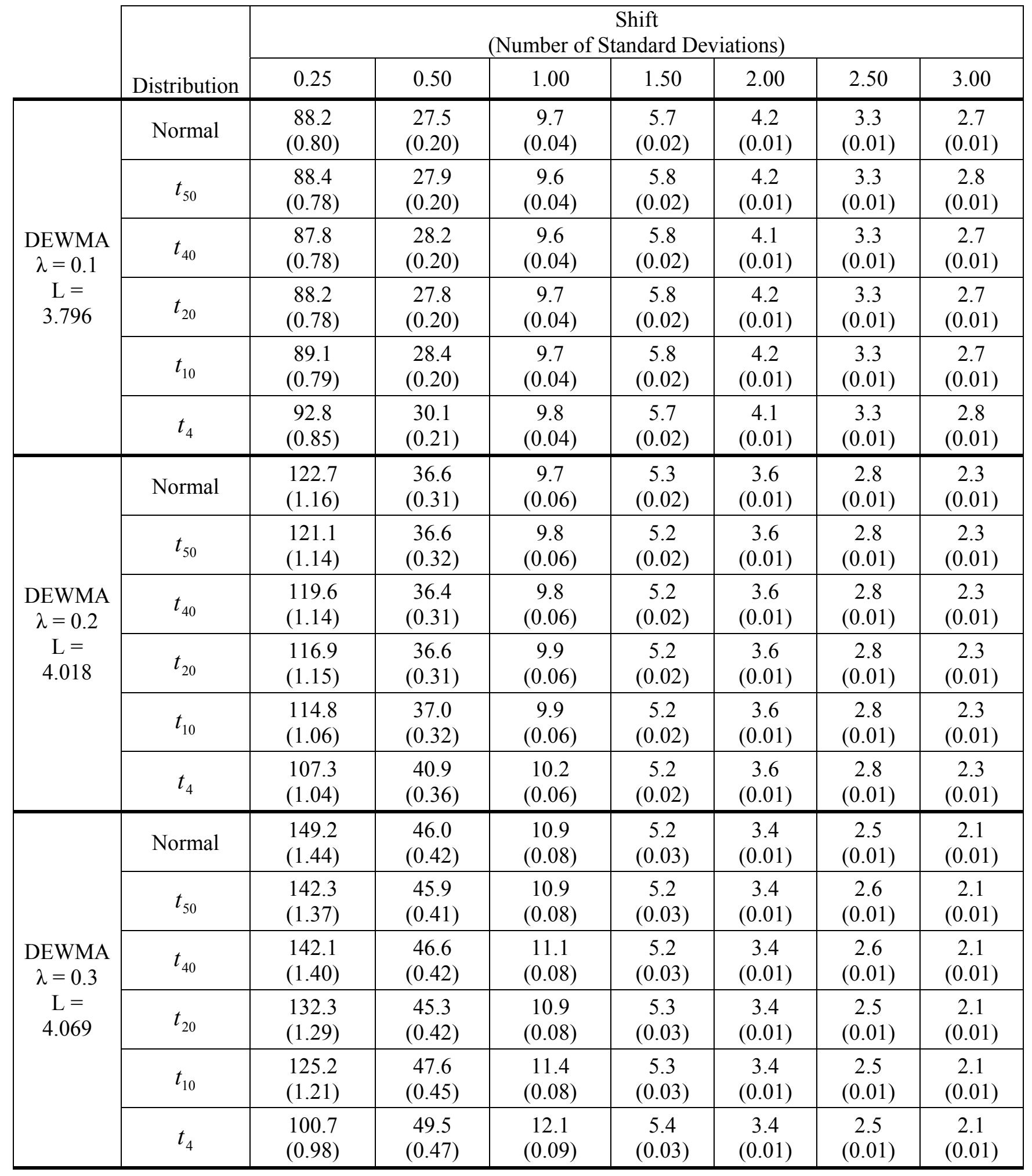


SAAD SAEED ALKAHTANI

Table 6 (continued): Out-of-control ARL's for the DEWMA Control Charts for Normal and Various t Distributions

\begin{tabular}{|c|c|c|c|c|c|c|c|c|}
\hline & \multirow[b]{2}{*}{ Distribution } & \multicolumn{7}{|c|}{$\begin{array}{c}\text { Shift } \\
\text { (Number of Standard Deviations) }\end{array}$} \\
\hline & & 0.25 & 0.50 & 1.00 & 1.50 & 2.00 & 2.50 & 3.00 \\
\hline \multirow{6}{*}{$\begin{array}{c}\text { DEWMA } \\
\lambda=0.4 \\
\mathrm{~L}= \\
4.059\end{array}$} & Normal & $\begin{array}{l}175.7 \\
(1.69)\end{array}$ & $\begin{array}{c}58.3 \\
(0.56)\end{array}$ & $\begin{array}{c}12.9 \\
(0.10)\end{array}$ & $\begin{array}{c}5.4 \\
(0.03)\end{array}$ & $\begin{array}{c}3.4 \\
(0.02)\end{array}$ & $\begin{array}{c}2.5 \\
(0.01)\end{array}$ & $\begin{array}{c}1.9 \\
(0.01)\end{array}$ \\
\hline & $t_{50}$ & $\begin{array}{l}163.7 \\
(1.62) \\
\end{array}$ & $\begin{array}{c}56.1 \\
(0.54) \\
\end{array}$ & $\begin{array}{c}12.8 \\
(0.10) \\
\end{array}$ & $\begin{array}{c}5.5 \\
(0.03) \\
\end{array}$ & $\begin{array}{c}3.4 \\
(0.02) \\
\end{array}$ & $\begin{array}{c}2.4 \\
(0.01)\end{array}$ & $\begin{array}{c}2.0 \\
(0.01)\end{array}$ \\
\hline & $t_{40}$ & $\begin{array}{l}164.9 \\
(1.62)\end{array}$ & $\begin{array}{c}58.3 \\
(0.57)\end{array}$ & $\begin{array}{c}12.8 \\
(0.10)\end{array}$ & $\begin{array}{c}5.5 \\
(0.03)\end{array}$ & $\begin{array}{c}3.4 \\
(0.02)\end{array}$ & $\begin{array}{c}2.4 \\
(0.01)\end{array}$ & $\begin{array}{c}1.9 \\
(0.02)\end{array}$ \\
\hline & $t_{20}$ & $\begin{array}{l}146.9 \\
(1.45)\end{array}$ & $\begin{array}{l}58.0 \\
(0.55)\end{array}$ & $\begin{array}{c}12.9 \\
(0.10)\end{array}$ & $\begin{array}{c}5.5 \\
(0.03)\end{array}$ & $\begin{array}{c}3.4 \\
(0.02)\end{array}$ & $\begin{array}{c}2.4 \\
(0.01)\end{array}$ & $\begin{array}{c}2.0 \\
(0.01)\end{array}$ \\
\hline & $t_{10}$ & $\begin{array}{l}130.0 \\
(1.29)\end{array}$ & $\begin{array}{c}55.2 \\
(0.52) \\
\end{array}$ & $\begin{array}{l}13.0 \\
(0.10)\end{array}$ & $\begin{array}{c}5.5 \\
(0.03) \\
\end{array}$ & $\begin{array}{c}3.4 \\
(0.02) \\
\end{array}$ & $\begin{array}{c}2.4 \\
(0.01) \\
\end{array}$ & $\begin{array}{c}1.9 \\
(0.01)\end{array}$ \\
\hline & $t_{4}$ & $\begin{array}{c}93.0 \\
(0.90)\end{array}$ & $\begin{array}{c}54.6 \\
(0.53)\end{array}$ & $\begin{array}{c}15.2 \\
(0.13)\end{array}$ & $\begin{array}{c}5.8 \\
(0.04)\end{array}$ & $\begin{array}{c}3.4 \\
(0.02)\end{array}$ & $\begin{array}{c}2.4 \\
(0.01)\end{array}$ & $\begin{array}{c}2.0 \\
(0.01)\end{array}$ \\
\hline \multirow{6}{*}{$\begin{array}{c}\text { DEWMA } \\
\lambda=0.5 \\
\mathrm{~L}= \\
3.991\end{array}$} & Normal & $\begin{array}{l}191.6 \\
(1.95)\end{array}$ & $\begin{array}{c}70.8 \\
(0.69) \\
\end{array}$ & $\begin{array}{c}15.2 \\
(0.13)\end{array}$ & $\begin{array}{c}6.0 \\
(0.04) \\
\end{array}$ & $\begin{array}{c}3.4 \\
(0.02) \\
\end{array}$ & $\begin{array}{c}2.4 \\
(0.01) \\
\end{array}$ & $\begin{array}{c}1.8 \\
(0.01) \\
\end{array}$ \\
\hline & $t_{50}$ & $\begin{array}{l}173.0 \\
(1.75)\end{array}$ & $\begin{array}{c}68.0 \\
(0.65)\end{array}$ & $\begin{array}{c}15.6 \\
(0.13)\end{array}$ & $\begin{array}{c}6.0 \\
(0.04)\end{array}$ & $\begin{array}{c}3.4 \\
(0.02)\end{array}$ & $\begin{array}{c}2.4 \\
(0.01)\end{array}$ & $\begin{array}{c}1.9 \\
(0.01)\end{array}$ \\
\hline & $t_{40}$ & $\begin{array}{l}169.7 \\
(1.67)\end{array}$ & $\begin{array}{c}69.9 \\
(0.68) \\
\end{array}$ & $\begin{array}{c}15.3 \\
(0.13)\end{array}$ & $\begin{array}{c}6.1 \\
(0.04) \\
\end{array}$ & $\begin{array}{c}3.4 \\
(0.02) \\
\end{array}$ & $\begin{array}{c}2.4 \\
(0.01) \\
\end{array}$ & $\begin{array}{c}1.8 \\
(0.01)\end{array}$ \\
\hline & $t_{20}$ & $\begin{array}{l}158.7 \\
(1.57)\end{array}$ & $\begin{array}{c}66.1 \\
(0.65) \\
\end{array}$ & $\begin{array}{c}15.6 \\
(0.13) \\
\end{array}$ & $\begin{array}{c}6.1 \\
(0.04) \\
\end{array}$ & $\begin{array}{c}3.4 \\
(0.02) \\
\end{array}$ & $\begin{array}{c}2.4 \\
(0.01)\end{array}$ & $\begin{array}{c}1.9 \\
(0.01)\end{array}$ \\
\hline & $t_{10}$ & $\begin{array}{l}125.8 \\
(1.22)\end{array}$ & $\begin{array}{c}61.7 \\
(0.60)\end{array}$ & $\begin{array}{c}15.8 \\
(0.14)\end{array}$ & $\begin{array}{c}6.2 \\
(0.04)\end{array}$ & $\begin{array}{c}3.5 \\
(0.02)\end{array}$ & $\begin{array}{c}2.4 \\
(0.01)\end{array}$ & $\begin{array}{c}1.9 \\
(0.01)\end{array}$ \\
\hline & $t_{4}$ & $\begin{array}{c}85.7 \\
(0.86)\end{array}$ & $\begin{array}{c}57.2 \\
(0.56)\end{array}$ & $\begin{array}{c}18.3 \\
(0.17)\end{array}$ & $\begin{array}{c}6.8 \\
(0.05)\end{array}$ & $\begin{array}{c}3.4 \\
(0.02)\end{array}$ & $\begin{array}{c}2.4 \\
(0.01)\end{array}$ & $\begin{array}{c}1.8 \\
(0.01)\end{array}$ \\
\hline
\end{tabular}




\section{Appendix A}

To obtain the DEWMA statistic $z_{i}$ defined in equation (1.5), repeated substitutions were applied to equations in (1.4) to obtain $y_{i}$ and $z_{i}$ rewritten as:

$$
\begin{aligned}
y_{i} & =\lambda x_{i}+(1-\lambda) y_{i-1} \\
& =\lambda \sum_{j=0}^{i-1}(1-\lambda)^{j} x_{i-j}+(1-\lambda)^{i} y_{0}
\end{aligned}
$$

and

$$
\begin{aligned}
z_{i} & =\lambda y_{i}+(1-\lambda) z_{i-1} \\
& =\lambda \sum_{j=0}^{i-1}(1-\lambda)^{j} y_{i-j}+(1-\lambda)^{i} z_{0}
\end{aligned}
$$

Substituting (A1) into (A2) results in:

$$
\begin{aligned}
z_{i} & =\lambda \sum_{j=0}^{i-1}(1-\lambda)^{j}\left[\lambda \sum_{k=0}^{i-j-1}(1-\lambda)^{k} x_{i-j-k}+(1-\lambda)^{i-j} y_{0}\right]+(1-\lambda)^{i} z_{0} \\
& =\lambda^{2} \sum_{j=0}^{i-1}(1-\lambda)^{j} \sum_{k=0}^{i-j-1}(1-\lambda)^{k} x_{i-j-k}+i \lambda(1-\lambda)^{i} y_{0}+(1-\lambda)^{i} z_{0} \\
& =\lambda^{2} \sum_{j=0}^{i-1}(1-\lambda)^{j} \sum_{l=1}^{i-j}(1-\lambda)^{i-j-l} x_{l}+i \lambda(1-\lambda)^{i} y_{0}+(1-\lambda)^{i} z_{0} \\
& =\lambda^{2} \sum_{l=1}^{i}(i-l+1)(1-\lambda)^{i-l} x_{l}+i \lambda(1-\lambda)^{i} y_{0}+(1-\lambda)^{i} z_{0}
\end{aligned}
$$

Replacing $l$ with $j,(\mathrm{~A} 3)$ is:

$$
\mathbf{z}_{i}=\lambda^{2} \sum_{j=1}^{i}(i-j+1)(1-\lambda)^{i-j} \mathbf{x}_{j}+i \lambda(1-\lambda)^{i} \mathbf{y}_{0}+(1-\lambda)^{i} \mathbf{z}_{0}
$$

where it was assumed, without loss of generality, that $y_{0}=z_{0}=\mu_{0}$.

The following quantities were presented by Gradshteyn and Ryzhik (1979).

For $a \neq 0$

$$
\sum_{k=1}^{n} k a^{k}=\frac{a\left(1-a^{n}\right)}{(1-a)^{2}}-\frac{n a^{n+1}}{1-a}
$$

and

$$
\sum_{k=1}^{n} k^{2} a^{k}=\frac{a+a^{2}-(n+1)^{2} a^{n+1}+\left(2 n^{2}+2 n-1\right) a^{n+2}-n^{2} a^{n+3}}{(1-a)^{3}}
$$

are needed to prove the quations (1.6) and (1.7). 


\section{SAAD SAEED ALKAHTANI}

Equation (1.6) Proof

$$
\text { Appendix A (continued) }
$$

$$
\begin{aligned}
\mu_{z_{i}} & =E\left(z_{i}\right)=E\left[\lambda^{2} \sum_{j=1}^{i}(i-j+1)(1-\lambda)^{i-j} x_{j}+i \lambda(1-\lambda)^{i} y_{0}+(1-\lambda)^{i} z_{0}\right] \\
& =\lambda^{2} \sum_{j=1}^{i}(i-j+1)(1-\lambda)^{i-j} E\left(x_{j}\right)+i \lambda(1-\lambda)^{i} E\left(y_{0}\right)+(1-\lambda)^{i} E\left(z_{0}\right) \\
& =\frac{\lambda^{2}}{1-\lambda} \sum_{j=1}^{i}(i-j+1)(1-\lambda)^{i-j+1} \mu_{0}+i \lambda(1-\lambda)^{i} \mu_{0}+(1-\lambda)^{i} \mu_{0}
\end{aligned}
$$

Letting $k=i-j+1$ in the first term

$$
\mu_{z_{i}}=\frac{\lambda^{2}}{1-\lambda} \sum_{k=1}^{i} k(1-\lambda)^{k} \mu_{0}+i \lambda(1-\lambda)^{i} \mu_{0}+(1-\lambda)^{i} \mu_{0}
$$

and applying equation (A.5) to the first term with $a=1-\lambda$ and $n=i$ results in

$$
\begin{aligned}
\mu_{z_{i}} & =\frac{\lambda^{2}}{1-\lambda}\left[\frac{(1-\lambda)\left[1-(1-\lambda)^{i}\right]}{[1-(1-\lambda)]^{2}}-\frac{i(1-\lambda)^{i+1}}{1-(1-\lambda)}\right] \mu_{0}+i \lambda(1-\lambda)^{i} \mu_{o}+(1-\lambda)^{i} \mu_{o} \\
& =\lambda^{2}\left[\frac{1-(1-\lambda)^{i}}{\lambda^{2}}-\frac{i(1-\lambda)^{i}}{\lambda}\right] \mu_{0}+i \lambda(1-\lambda)^{i} \mu_{0}+(1-\lambda)^{i} \mu_{0} \\
& =\left[1-(1-\lambda)^{i}-i \lambda(1-\lambda)^{i}+i \lambda(1-\lambda)^{i}+(1-\lambda)^{i}\right] \mu_{0} \\
& =\mu_{0}
\end{aligned}
$$

Equation (1.7) Proof

$$
\begin{aligned}
\sigma_{z_{i}}^{2} & =\operatorname{Var}\left[z_{i}\right]=\operatorname{Var}\left[\lambda^{2} \sum_{j=1}^{i}(i-j+1)(1-\lambda)^{i-j} x_{j}+i \lambda(1-\lambda)^{i} y_{0}+(1-\lambda)^{i} z_{0}\right] \\
& =\lambda^{4} \sum_{j=1}^{i}(i-j+1)^{2}\left[(1-\lambda)^{2}\right]^{i-j} \operatorname{Var}\left(x_{j}\right)+0 \\
& =\frac{\lambda^{4}}{(1-\lambda)^{2}} \sum_{j=1}^{i}(i-j+1)^{2}\left[(1-\lambda)^{2}\right]^{i-j+1} \sigma^{2}
\end{aligned}
$$

Applying equation (A.6) with $a=(1-\lambda)^{2}, k=i-j+1$ and $n=i$, results in:

$$
\begin{aligned}
\sigma_{z_{i}}^{2} & =\frac{\lambda^{4}}{(1-\lambda)^{2}} \frac{(1-\lambda)^{2}+(1-\lambda)^{4}-(i+1)^{2}(1-\lambda)^{2 i+2}+\left(2 i^{2}+2 i-1\right)(1-\lambda)^{2 i+4}-i^{2}(1-\lambda)^{2 i+6}}{\left[1-(1-\lambda)^{2}\right]^{3}} \sigma^{2} \\
& =\lambda^{4} \frac{1+(1-\lambda)^{2}-(i+1)^{2}(1-\lambda)^{2 i}+\left(2 i^{2}+2 i-1\right)(1-\lambda)^{2 i+2}-i^{2}(1-\lambda)^{2 i+4}}{\left[1-(1-\lambda)^{2}\right]^{3}} \sigma^{2}
\end{aligned}
$$

